\title{
Hydrocortisone and Indomethacin Negatively Modulate EGF-R Signaling in Human Fetal Intestine
}

\author{
RISTO KAJANNE, SIRPA LEPPÄ, PÄIVI LUUKKAINEN, JARKKO USTINOV, ALEXANDRA THIEL, ARI RISTIMÄKI, \\ AND PÄIVI J. MIETTINEN
}

\author{
Molecular Cancer Biology Program [R.K., S.L.], Genome-Scale Biology Research Program [A.T., A.R.], University of Helsinki, \\ FIN-00014, Finland; Hospital for Children and Adolescents [P.L., J.U., P.J.M.], Department of Oncology [S.L.], Pathology/Huslab [A.R.], \\ Helsinki University Central Hospital, FIN-00029, Finland
}

\begin{abstract}
Concomitant use of hydrocortisone and the nonspecific cyclo-oxygenase (COX)-inhibitor indomethacin increases the risk for intestinal perforations in preterm infants. We determined whether this was associated with insufficient epidermal growth factor receptor (EGF-R) signaling. We tested the effect of EGF, hydrocortisone, and indomethacin on its activation, cell proliferation and migration, COX-2 expression, and prostaglandin $\mathrm{E}_{2}\left(\mathrm{PGE}_{2}\right)$ production. Human small intestine epithelial cell line FHsInt74 and EGF-Rdeficient mice [EGF-R (-I-)] were used as models. The data revealed that EGF-R signaling had a bimodal positive effect on fetal enterocyte: 1) it increased cell proliferation and migration synergistically with hydrocortisone and 2) up-regulated COX-2 mRNA expression and subsequent $\mathrm{PGE}_{2}$ production. Correlating with this, $\mathrm{COX}-2$ protein expression was down-regulated in EGF-R (-l-) intestine. Despite a positive effect on cell proliferation with EGF, hydrocortisone blunted the stimulatory effect of EGF on COX-2 expression and $\mathrm{PGE}_{2}$ production. Addition of indomethacin even further inhibited the EGF-stimulated $\mathrm{PGE}_{2}$ synthesis. The data suggest that concomitant use of indomethacin and hydrocortisone on preterm infants, who physiologically synthesize only low levels of EGF-R ligands, may lead to intestinal problems related to failure in cytoprotective and regenerative events. (Pediatr Res 62: 570-575, 2007)
\end{abstract}

$\mathrm{D}$ uring pregnancy, fetal mucosal surfaces are constantly in contact with amniotic fluid rich in growth factors and hormones. After a preterm delivery, the infant is deprived of these substances and exposed to a stressful environment. Due to immaturity of organ systems, preterm infants are at risk to suffer from diseases associated with immaturity, e.g. respiratory distress syndrome (RDS), patent ductus arteriosus (PDA), and intestinal perforations (including necrotizing enterocolitis). In clinical trials, $\mathrm{HC}$ has been used to improve the outcome of preterm infants (1). In preterm infants, indomethacin (Ind) an inhibitor of both cyclo-oxygenase (COX)-1 and COX-2, is used to treat PDA. Side-effects related to Ind treatment include gastric lesions and, lately also spontaneous intestinal perforation (SIP) (2). Identification of cellular pathways leading to these events is critical. Data concerning the

Received February 9, 2007; accepted June 28, 2007.

Correspondence: Päivi J. Miettinen, M.D., Ph.D., Laboratory for Hospital for Children and Adolescents, Biomedicum Helsinki, Room B428b, P.O. Box 63 (Haartmaninkatu 8), FIN-00014 University of Helsinki, Finland; e-mail: paivi.miettinen@ helsinki.fi

The Sigrid Jusélius Foundation, the Finnish Medical Foundation, Finnish Cancer Societies, University of Helsinki, and the Helsinki University Central Hospital Research grant (EVO) supported this study. role of Ind treatment and other potential factors in the development of gastric lesions in preterm infants on a cellular level is scarce. The role of glucocorticoids in this context remains unclear. Recently, a harmful synergism hypothesis involving both early postnatal Ind and glucocorticoids in the etiology of SIP was presented (2). When used alone to close PDA, Ind does not increase the risk for NEC (3), although studies with contradicting results exist $(4,5)$.

Epidermal growth factor (EGF) receptor ligands (amphiregulin, betacellulin, EGF, epigen, epiregulin, heparin-binding EGF, and TGF- $\alpha$ ) bind to and activate their receptor, EGF-R. EGF-R is expressed throughout the fetal and neonatal gastrointestinal tract and is involved in enterocyte proliferation, migration, differentiation and protection from apoptosis $(6,7)$. Its targeted inactivation leads to impaired epithelial development of the gastrointestinal tract and NEC-like lesions (8). Amniotic fluid is rich in EGF and after birth, maternal milk is the major source of EGF. In rodent and rabbit models, EGF supplementation has been shown to reduce the incidence and severity of NEC (9). Interestingly, deficient local mucosal protection has been suggested to contribute to the pathophysiology of NEC/SIP and EGF has been shown to improve barrier function (10). Prostaglandins, which are synthesized from arachidonic acid by cyclo-oxygenases (COX), are important in the maintenance of mucosal integrity. Since EGF-R and COX-2 signaling have been linked together in oncology, we analyzed whether this occurs also during human intestinal development. In addition, we studied whether any biologic mechanism for the negative interplay between them, HC and Ind could be found in human fetal intestine.

\section{MATERIALS AND METHODS}

Cell culture. Human small intestine epithelial FHs74Int cells (ATCC, Manassas, VA; CCL-241) were cultured in a humidified $5 \% \mathrm{CO}_{2}$ atmosphere at $37^{\circ} \mathrm{C}$ in Dulbecco's modified Eagle's medium (DMEM) supplemented with $10 \%$ FCS, $2 \mathrm{mM}$ glutamine, $100 \mathrm{unit} / \mathrm{mL}$ penicillin, $100 \mu \mathrm{g} / \mathrm{mL}$ streptomycin, nonessential amino acids, sodium pyruvate $(0.5 \mathrm{mM})$, oxaloacetic acid (1 $\mathrm{mM})$, and insulin $(0.2$ units $/ \mathrm{mL})$. Cells were stimulated with $20 \mathrm{ng} / \mathrm{mL}$ EGF (Sigma Chemical Co., St. Louis, MO), 50 ng/mL HC (Pfizer, New York, NY) or $10 \mu \mathrm{M}$ prostaglandin $\mathrm{E}_{2}\left(\mathrm{PGE}_{2}\right)$ (Cayman Chemical, Ann Arbor, $\left.\mathrm{MI}\right)$.

Abbreviations: COX, cyclo-oxygenase; EGF, epidermal growth factor; HC, hydrocortisone; Ind, indomethacin; NEC, necrotizing enterocolitis; PDA, patent ductus arteriosus; $\mathbf{P G E}$, prostaglandin $\mathrm{E}_{2} ; \mathbf{S I P}$, spontaneous intestinal perforation 
EGF-R-inhibitor ZD1839 (10 $\mu \mathrm{M}$; AstraZeneca, London, UK), Mek-inhibitor PD98059 (20 $\mu \mathrm{M}$; Calbiochem, San Diego, CA), PI3 K-inhibitor LY294002 (20 $\mu \mathrm{M}$; Calbiochem), and COX-inhibitor Ind (50 $\mu \mathrm{M}$, Dumex-Alpharma (Copenhagen, Denmark) were added to the cultures $1 \mathrm{~h}$ before stimulants. UV light $\left(30 \mathrm{~J} / \mathrm{m}^{2}\right)$ was used as a positive control treatment for the activation of p38 and Jnk MAPKs. For proliferation analyses, FHs74Int cells were plated $(25,000$ cells/24 wells) in duplicate wells and cultured for 3,6 , or $9 \mathrm{~d}$. Cell number was determined after trypsinization using Burker chamber and light microscopy.

Tissue samples. Murine gut samples were obtained from EGF-R (-l-) and wt mice at embryonic d 17 (E17) and postnatal d 3 (P3) (8). Human fetal second trimester intestines were collected via legal abortions. Studies were approved by the Helsinki University Animal Ethics Committee and the Ethical Committee of the Helsinki Maternity Clinic. Samples for immunohistochemistry were fixed in $4 \%$ paraformaldehyde and embedded in paraffin as routinely. Mouse intestine for Western analysis was trimmed from the abdominal fat and viscera, divided into duodenum, jejunum, ileum, and colon, snap-frozen, and stored at $-70^{\circ} \mathrm{C}$ until further analysis.

Immunofluorescence staining. FHs74Int cells were fixed in 4\% paraformaldehyde (PFA). EGF-R-phospho-specific antibodies (\#44-788G, \#44-790, \#44-974G; Biosource International, Camarillo, CA) and Texas-Redconjugated secondary antibodies (Jackson Laboratories, Bar Harbor, ME) were used. Actin filaments were stained with TRITC-conjugated phalloidin (Sigma Chemical Co.).

Western analysis and immunoprecipitation. FHs74Int cells were lysed in SDS sample buffer and sonicated. Protein concentration was measured as described (11). Briefly, the samples were blotted onto Whatman 3MM membrane, and proteins stained with Coomassie brilliant blue. BSA was used to create a standard curve to which each sample dot was compared. A good linearity in protein amounts was obtained between 0.25 and $10 \mu \mathrm{g} / \mu \mathrm{L}$. An equal amount (50 $\mu \mathrm{g} / \mathrm{lane})$ of protein was separated in a 10\% SDS-PAGE gel and transferred onto nitrocellulose membrane (Bio-Rad, Hercules, CA) by electroblotting. Immunoblotting was performed using antibodies against phospho-Erk and -Jnk (V803A, V7932; Promega, Madison, WI), Erk1/2 and p21 (sc-154, sc-397; Santa Cruz Biochemicals, Santa Cruz, CA), phosphoAkt and Akt (9271S, 9272; Cell Signaling Technology, Beverly, MA), phospho-p38 and p38 (9211S, 9212; Cell Signaling Technology), phosphoEGF-R, cyclinD1 (DCS6, NeoMarkers, Lab Vision Products, Thermo Fisher Scientific, Fremont, CA), COX-2 (\#160126; Cayman Chemical) and $\alpha$-tubulin (\#518, Sigma Chemical Co.). Horseradish peroxidase-conjugated secondary antibodies (Jackson Laboratories) and enhanced chemiluminescence protocol (Super Signal, Pierce, Rockford, IL) were used.

For immunoprecipitation, cells were lysed in RIPA lysis buffer. Supernatants were incubated with protein A Sepharose beads (Amersham, Little Chalfont, Buckinghamshire, UK) and appropriate antibodies for overnight at $4^{\circ} \mathrm{C}$. After washing in RIPA buffer, the absorbed complexes were removed from the beads by heating in SDS sample buffer and subjected to Western analysis. All experiments were done in triplicates. Tubulin, Erk, or p38 expression was used as loading controls.

Immunohistochemistry. Paraffin-sections $(5 \mu \mathrm{m})$ were deparaffinized. For antigen unmasking, the slides were microwaved in $10 \mathrm{mM}$ citrate-buffer (for COX-2 detection) or Proteinase K (Ready-to-use Proteinase K; Dako Cytomation, Glostrup, Denmark) treated (for EGF-R detection). Immunostaining was performed with COX-2 or EGF-R antibodies (\#sc-03; Santa Cruz Biochemicals) and biotinylated goat anti-rabbit immunoglobulins (Vector Laboratories, Burlingame, CA). Immunoreactivity was visualized by avidin-biotin peroxidase complex solution (Vectastain ABComplex, Vector Laboratories) and 3-amino-9-ethylcarbazole (Lab Vision). Counterstaining was performed with Mayer's hemalum (Merck, Darmstadt, Germany). Nonimmune rabbit serum served as a negative control.

Real-time-PCR. Total RNA was isolated using RNeasy Mini Kit (QIAGEN, Valencia, CA) and converted to cDNA using SuperScript First-Strand Synthesis System (Invitrogen, Carlsbad, CA) with random hexamers. Realtime PCR reactions were performed with the Gene Amp 5700 Sequence detection system (Applied Biosystems, Foster City, CA). Human 18S rRNA served as an endogenous control. Each sample was measured in triplicates, and data analyzed by the delta-delta method for comparing relative expression

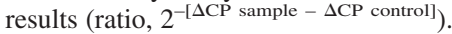

$P G E_{2}$ ELISA. PGE $_{2}$ concentration in the conditioned culture medium was analyzed using enzyme immunoassay according to the manufacturer's protocol (Cayman Chemical). Measurements were done from five consecutive experiments in duplicates for each stimulant.

Migration assay. Cell migration was analyzed using Transwell chambers with gelatin-precoated filters ( $8 \mu \mathrm{m}$ pores) in 24-well tissue culture plates (BD Biosciences, San Jose, CA). Cells $\left(5 \times 10^{4}\right)$ were added to the upper side and the inhibitors and stimulants to the lower side of the membrane. After $36 \mathrm{~h}$, the cells were fixed (2\% PFA) and noninvaded cells were removed by wiping the upper side of the membrane. Invaded cells were stained using crystal violet, photographed, and quantified by dissolving crystal violet staining to $10 \%$ acetic acid and measuring the absorbance using Multiskan EX photometric absorbance reader (Thermo Fisher Scientific, Waltham, MA). Experiments were done in triplicates and repeated five times.

Statistical analysis. All data are expressed as the mean \pm SEM. Unpaired $t$ test was used for significance calculations. A value of $p<0.05$ was used as the limit for statistical significance.

\section{RESULTS}

EGF-R signaling in FHs74Int cells. FHs74Int cells, which are derived from a first-trimester human fetal small intestine, were chosen as an in vitro model to study the interactions between EGF-R signaling, glucocorticoids, and prostaglandins. We first confirmed that they expressed EGF-R. As seen in Figure 1A, EGF-R was mainly located on the cell membrane and became phosphorylated upon EGF stimulation. This led to activation of the downstream signaling routes, namely the MAPK (pErk) and PI-3 kinase pathways (pAkt) (Fig. 1B). The specific EGF-R inhibitor ZD1839 blocked EGF-R phosphorylation and down-regulated both of the signaling pathways to basal levels. EGF had no effect on the phosphorylation of Jnk or p38 (Fig. 1B). UV-treatment was used as a positive control for their activation. This effect was not inhibited by ZD1839.

Combination of EGF and HC increases proliferation. EGF ligands have been shown to increase enterocyte proliferation (12), and coinciding with this, the proliferative index is low in EGF-R (-/-) jejunum (8). To explore the interaction of EGF, glucocorticoids, and prostaglandins on enterocyte proliferation, FHs74Int cells were grown in DMEM in their presence and cell numbers counted during a 9-d culture period. This time frame was chosen to mimic the in vivo intrauterine conditions. In this model, the effect of EGF on enterocyte proliferation was time-dependent (Fig. 2). At d 3, cell number was increased by $20 \%$ and further by $80 \%$ at $\mathrm{d} 9$. This stimulatory effect was completely prevented by ZD1839. HC, Ind, or $\mathrm{PGE}_{2}$ alone had no significant effect on cell proliferation. However, when HC was combined with EGF, they increased cell proliferation by $131 \pm 7 \%$ (d 6) and by $162 \pm 17 \%$ (d 9), i.e. more than EGF did alone. Again, ZD1839, but not Ind, inhibited this. EGF-induced cell proliferation was also inhibited by the MAPK- and PI3-kinase inhibitors. Cell apoptosis was determined by propidium iodine staining and FACS analysis or caspase- 3 immunohistochemistry after cultivation in
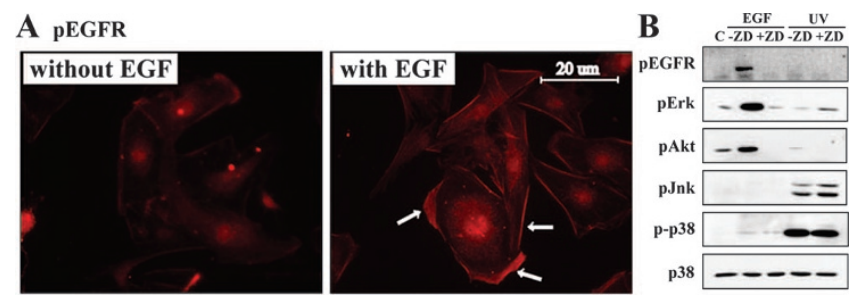

Figure 1. EGF-induced activation of EGF-R signaling pathways in FHs74Int cells. (A) Stimulation with EGF resulted in the appearance of phosphorylated EGF-R in the cell membrane (arrows). (B) FHs74Int cells were treated as indicated and effects of EGF were controlled in the presence of ZD1839. UV-treatment was used as a positive control for Jnk and p38 activation. 


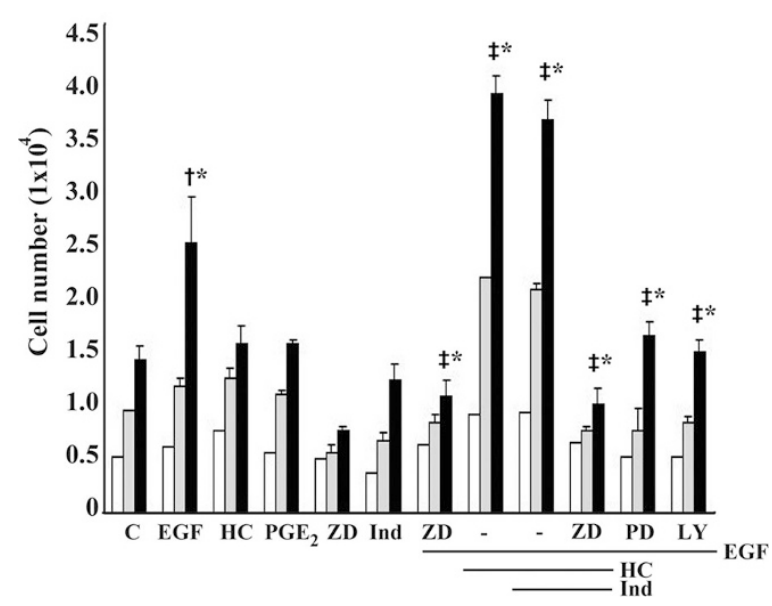

Figure 2. HC potentiated EGF-induced proliferation of FHs74Int cells. Fhs74Int cells were cultured and cell numbers were counted on d 3 (white bars), 6 (shaded bars) and 9 (black bars). EGF alone and in combination with HC synergistically increased cell proliferation, whereas ZD1839 inhibited these effects. PD and LY inhibited EGF-induced cell proliferation. $†$ Untreated vs EGF-treated; \$>EGF $v s \mathrm{EGF}+\mathrm{HC}, \mathrm{EGF}+\mathrm{HC}+\mathrm{Ind}, \mathrm{EGF}+\mathrm{HC}+\mathrm{Ind}+\mathrm{ZD}$, $\mathrm{EGF}+\mathrm{PD}$, or $\mathrm{EGF}+\mathrm{LY} ; * p<0.05$.

serum-free media with or without EGF, $\mathrm{PGE}_{2}, \mathrm{HC}$, and Ind. Only Ind treatment induced apoptosis, but this was only at high concentrations (100 $\mu \mathrm{M}$; data not shown).

Next, we searched for molecular mechanisms explaining the potentiating effect of EGF and $\mathrm{HC}$ on cell proliferation and looked at whether HC could activate EGF-R. However, no increase in EGF-R phosphorylation was detectable after HC stimulation (Fig. $3 A$ ). Nor did $\mathrm{HC}$ have any effect on pErk or pAkt activities even when combined to EGF (Fig. 3A). It was recently reported that EGF-R-mediated enterocyte proliferation requires p21 expression through MAPK-dependent mechanisms (13). We analyzed whether $\mathrm{p} 21$ is also involved in proliferative synergism between EGF and HC. While stimulation of FHs74Int cells with EGF led to up-regulation of both p21 and cyclin D1 expression, this was not evident with HC (Fig. 3B).

COX-2 expression and EGF-R signaling. Although $\mathrm{PGE}_{2}$ had no significant effect on the growth of the FHsInt74 cells, inhibitor of its synthesis, Ind, has been associated with intestinal lesions in vivo (2). Ind inhibits both COX-1 and -2. As both COX-2 and EGF-R ligands have been implicated in intestinal physiology, we next explored whether their signaling pathways cross in fetal intestine. First, COX-2 expression

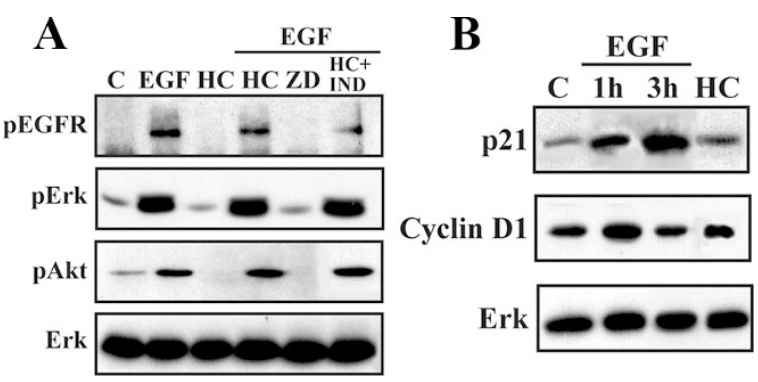

Figure 3. Activation of signal transduction pathways in FHs74Int cells by EGF and HC. (A) HC had no effect on EGF-R activation or EGF-induced Erkand Akt-activation. $(B)$ Expression of p21 and cyclinD1 was up-regulated by EGF, while HC had no effect on them. in the fetal intestine was analyzed. Human fetal small intestine and colon samples, 18-20 gestational weeks old, were stained with anti-COX-2. A strong COX-2 immunoreactivity was seen in the crypts in duodenum and jejunum extending to the lower one-third of the crypt-villous axis (Fig. 4). Only weakly stained cells were visible in the ileum. The COX-2 expression pattern resembled that of EGF-R (Fig. 4D). Interestingly, no COX-2 containing cells were detectable in the colon (data not shown), where EGF-R and its ligands are normally strongly expressed (6). Nonimmune sera gave no staining (Fig. 4E). We then analyzed COX-2 immunoreactivity in the EGF-R deficient mice. EGF-R (-/-) mice die during the first week of life due to epithelial immaturity and have short villi and spontaneous perforations in their intestine (8). A strong COX-2 immunoreactivity was detected in wild-type mouse jejunum at embryonic d 17 (E17) and postnatal d 3 (P3). It located to crypts and proximo distally in the villous axis (Fig. $5, A$ and $B$ ). Surprisingly, it was greatly reduced in the EGF-R $(-/-)$ jejunum both at E17 and P3 (Fig. 5, $C$ and $D$ ). These immunohistochemical results were also confirmed by measuring down-regulated COX-2 protein levels from EGF-R (-l-) mice fetal intestines with Western analysis (Fig. 5E). To conclude, the data suggest that intact EGF-R signaling is required for COX-2 expression.

Next, we performed quantitative RT-PCR to analyze whether COX-2 mRNA expression is regulated by EGF. For this purpose, FHs74Int cells were grown in serum-free media supplemented with EGF. As can be seen in Figure 6A, EGF up-regulated COX-2 mRNA levels by 25 - to 30 -fold $(p<0.01)$. This effect could be inhibited by pretreating the cells with inhibitors to both EGF-R, and its downstream kinases MAPK and PI3-K. Interestingly, EGF-induced COX-2 mRNA expression also was downregulated by $\mathrm{HC}(p<0.01)$, which alone had no significant effect on COX-2 transcription. To validate the changes in COX-2 mRNA expression, $\mathrm{PGE}_{2}$ production was measured by ELISA. Correlating with the induced COX-2 mRNA expression, EGF increased $\mathrm{PGE}_{2}$ production by $175 \%(p<0.01)$ (Fig. $\left.6 B\right)$. In

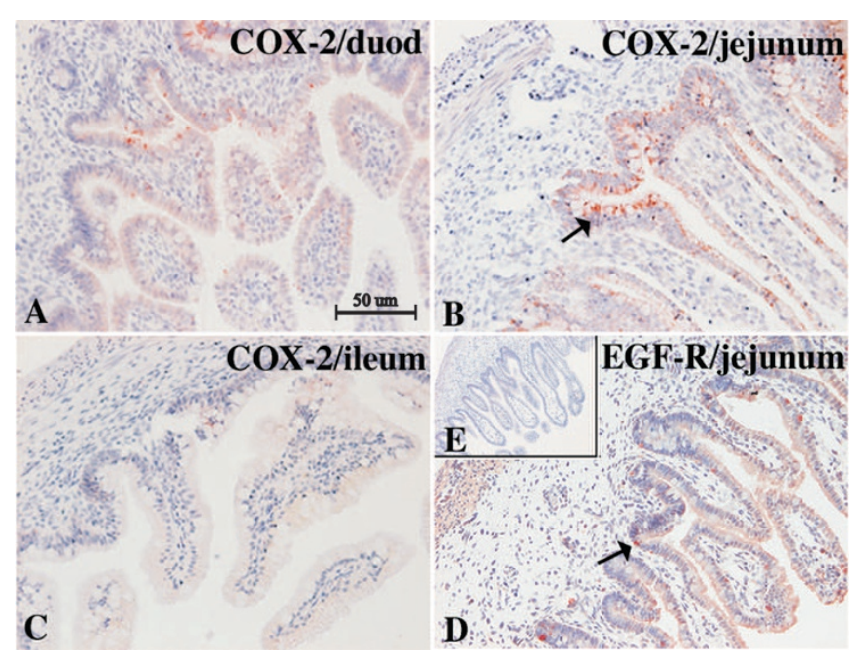

Figure 4. Immunostaining for COX-2 and EGF-R in human fetal small intestine. A strong COX-2 immunoreactivity (arrow) was seen in the lower crypt-villous axis of the duodenum $(A)$ and jejunum $(B)$, whereas only very few positive cells were visible in the ileum $(C)$. This staining pattern mimics the jejunal location of EGF-R $(D)$. Nonimmune serum was used as a negative control $(E)$. 
accordance with the PCR results, HC reduced EGF-induced $\mathrm{PGE}_{2}$ production by $40 \%(p<0.05)$, and Ind, EGF-R, MAPK and PI3-K inhibitors suppressed it to baseline. Interestingly, $\mathrm{PGE}_{2}$ stimulation of FHs74Int cells led to a rapid phosphorylation of EGF-R, Erk, and Akt, which was inhibited by ZD1839 (Fig. 6C). Thus, activation of both EGF-R and $\mathrm{PGE}_{2}$ pathways occurs synergistically in human fetal intestine.

Migration of FHs74Int cells. Enterocytes proliferate along one-third of the crypt-villus axis and then migrate toward the tip of the villous to substitute for mucosal damage and cell exfoliation. Since defects in this pathway might lead to intestinal perforation, we next analyzed whether cell migration is affected by various stimulants. In Transwell migration assay, addition of EGF, $\mathrm{HC}$ and $\mathrm{PGE}_{2}$ consistently $(p<0.05)$ increased FHs74Int cell migration during the 36-h culture period (Fig. 7A). This was not due to cell proliferation, inasmuch as EGF had no impact on cell numbers during a 3-9 culture (Fig. 2). Combination of EGF and HC doubled cell migration $(p<0.05)$, and the addition of Ind inhibited this by $15 \%(p<0.05)$, whereas ZD1839 completely prevented it $(p<0.05)$. The increase in migration correlated with EGFinduced transformation in cell phenotype toward a fibroblastlike appearance. This was visualized by a change from cuboidal cell shape with cortical actin expression into elongated cells with stress-fibers (Fig. $7 B$ ). The transition was most prominent when EGF and $\mathrm{HC}$ were used in combination, and blunted by the addition of Ind (Fig. 7B).

\section{DISCUSSION}

We sought to determine why concomitant use of $\mathrm{HC}$ and Ind increases the risk for intestinal perforations in preterm infants and whether this is associated with insufficient EGF-R signaling. Our results show that EGF and HC have synergistic mitogenic and migratory effects on human fetal enterocytes, which is also seen as a change toward a fibroblastoid phenotype. This has been also reported from ovarian epithelium, where combination of EGF and $\mathrm{HC}$ induced epithelial-to-mesenchymal transition (14). The nonspecific COX-inhibitor Ind slightly inhibited the EGFand HC-stimulated cell migration. Szabo (15) has described that Ind causes loss of actin stress fibers and inhibits EGF-R-mediated phosphorylation of focal adhesion kinase and tensin. These results agree with our observation of intestinal cells migratory phenotype. Previously, EGF and $\mathrm{HC}$ have been reported to affect variably cell proliferation in human fetal intestine $(16,17)$. In the F7Hs4Int cells, EGF-R-mediated signaling led to up-regulation of p21 and cyclinD1 correlating with the latest literature (13). The molecular basis for the growth promoting effect of $\mathrm{HC}$ and EGF combination remains open as no difference was seen in the EGF-R downstream signaling pathways. Nevertheless, it is EGF-R dependent since it was blocked by the EGF-R specific inhibitor. One possibility could be that HC leads to up-regulation of EGF-ligands or the number and affinity of EGF-R as has been previously suggested (14).

Hypoxia in the vessel wall is needed for the permanent closure of PDA after birth. In preterm infants, excess production of prostaglandins and nitric oxide relaxes smooth muscle cells and prevents local hypoxia to develop thus keeping the duct open (18). To overcome this, Ind is widely used in the treatment of PDA. Besides being a vasoconstrictor, it inhibits cell cycle arresting cells in G1 phase (19). In our study, Ind did not affect cell proliferation, but was apoptotic at high concentrations and impaired EGF- and HC-induced migration. Mucosal restitution is an early event to repair superficial injuries and may occur without cell proliferation (20). Besides being chemoattractants, EGF ligands affect cellular migration machinery (21). Thus, it is conceivable that dysregulation in EGF-R activation and concomitant Ind treatment could negatively modulate healing of spontaneous perforations.

Prostaglandins have important roles in intestinal physiology: they are cytoprotective, elevate villous labeling index (22), and their overproduction has been implicated in gastrointestinal malignancies. A link between the key enzyme in prostaglandin synthesis, COX-2, and EGF-R signaling pathways has been shown in many studies. In colon cancer and gastric epithelial cells, $\mathrm{PGE}_{2}$ phosphorylates EGF-R and induces cell proliferation (23). Vice versa, blocking the COX-2 pathway inhibits proliferation of pancreatic cancer cells, which is potentiated by simultaneous exposure to EGF-R inhibitors (24). In our study, EGF up-regulated COX-2 mRNA and $\mathrm{PGE}_{2}$ production, which, based on previous reports, may induce positive feedback on EGF-R signaling. Furthermore, HC blunted the EGF-induced COX-2 up-regulation, but alone had no significant effect on its transcription contrary to previous reports from other cell types (25). However, these studies have been done using a more potent glucocorticoid dexamethasone.

It is common knowledge that glucocorticoids and nonsteroidal anti-inflammatory drugs, including Ind, are ulcerogenic whereas EGF has historically been used in the treatment of gastric ulcers in Zollinger-Ellison syndrome (26). Effects of Ind are mediated through prostaglandin down-regulation and disturbed vascular circulation. Glucocorticoids, particularly dexamethasone, make gastric mucosal susceptible to ulceration by enhancing production of gastric acid and affecting prostaglandin synthetase and peroxidase. Interestingly, dexamethasone treatment leads to down-regulated EGF-R and TGF- $\alpha$ expression in muscularis mucosae and thinning of the ileal wall (27). EGF-R signaling, on the other hand, seems to be important for intestinal wall and adaptation processes since EGF-R transcription is up-regulated in smooth muscle layer after bowel resection (28). Taken together, combination of prostaglandin inhibition, glucocorticoid treatment, and insufficient EGF-R signaling could be detrimental to intestinal wall leading to perforations.

In accordance with the proliferative effect of EGF in FHs74Int cells, EGF-R activation enhances recovery from small bowel resection (SBR) (28). Adaptation of the premature gut to extrauterine conditions mimics SBR, i.e. development of taller villi, deeper crypts and enhanced enterocyte turnover (28). Moreover, problems seen with preterm infants resemble animal models of insufficient EGF-R signaling, i.e. deletion of EGF-R gene or down-regulation of its kinase activity (waved-2 mice or treatment with EGF-R-inhibitors). These mice have short intestinal villi, reduced cell proliferation, and subsequent development of NEC- 


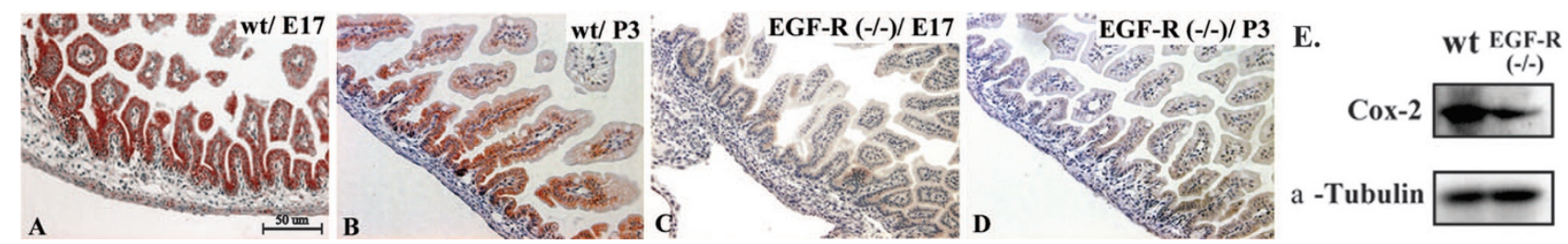

Figure 5. COX-2 immunostaining in wt and EGF-R (-I-) mouse small intestine. COX-2 immunoreactivity was very prominent (brown) in the wt jejunum at E17 $(A)$ and P3 $(B)$, whereas hardly any cells could be detected in the EGF-R $(-/-)$ jejunum $(B, D)$. Western analysis $(E)$ revealed that COX-2 protein levels were higher in the wt than in the EGF-R (-/-) intestine.

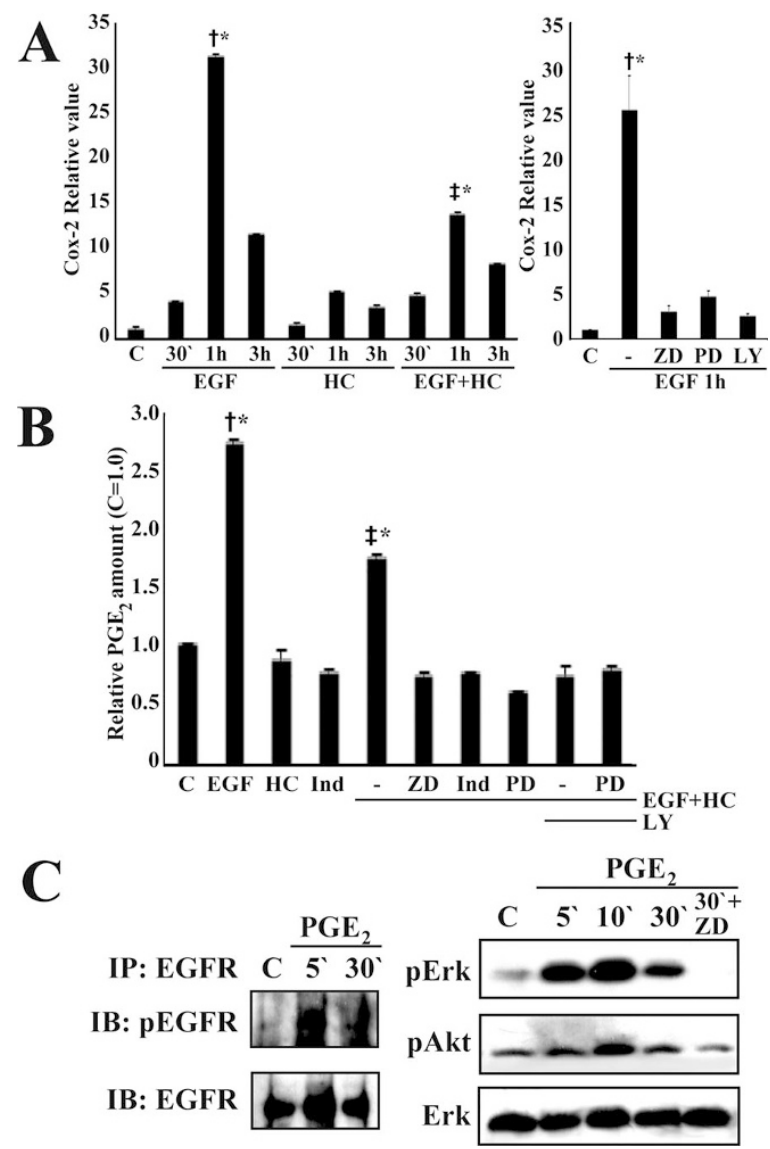

Figure 6. EGF up-regulated COX-2 mRNA and $\mathrm{PGE}_{2}$ protein levels. FHs74Int cells were grown as indicated without $(A)$ or with $(B)$ pretreatment with ZD, PD, and LY. (C) $\mathrm{PGE}_{2}$ leads to activation of EGF-R, Erk, and Akt pathways. †Untreated $v$ EGF-treated;

like lesions together with susceptibility to colitis and defective recovery from gut resection $(8,29)$. Clearly, EGF supplementation is beneficial to intestinal development, postnatal maturation, and adaptation $(30,31)$. It increases expression of anti-apoptotic proteins while suppressing pro-apoptotic proteins thus maintaining intestinal integrity (31). Furthermore, EGF improves intestinal barrier function by accelerating goblet cell maturation and mucin production and by normalizing the expression of tight junction proteins (10). This is crucial also in protection from NEC. Thus, EGF ligands might be useful for preterm infants. Indeed, EGF has been used incidentally in congenital microvillus atrophy (32), prevention of NEC (33), and pediatric short bowel syndrome (30). Association of EGF-R signaling to carcinogenesis has prevented routine EGF substitution. On the other hand, many events occurring during fetal development are activated as
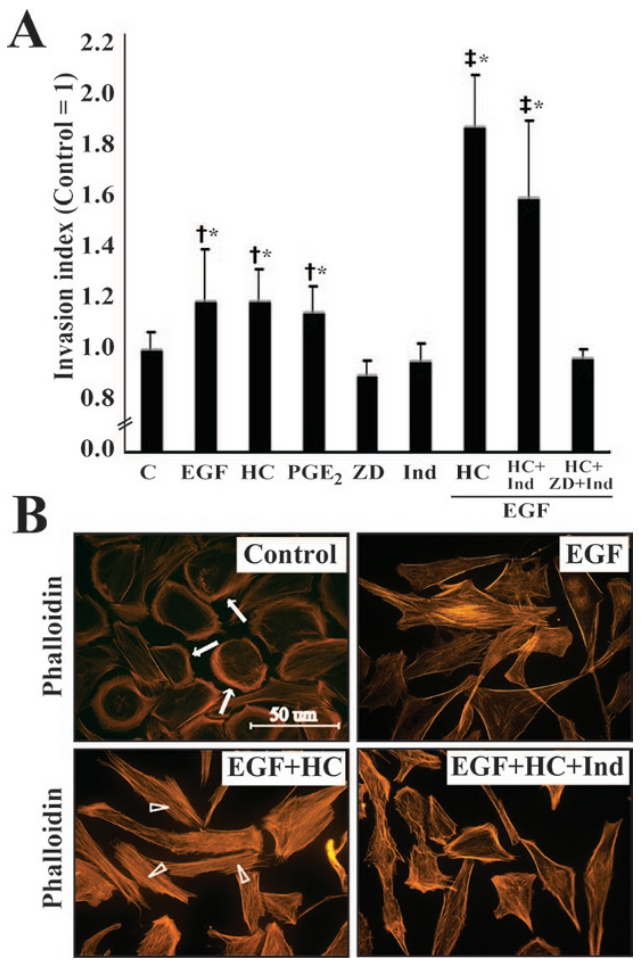

Figure 7. EGF and HC increased migration of FHs74Int cells. (A) FHs74Int cells were grown in Transwell chambers as indicated. Relative migration rates compared with untreated cells $(C$, control) are shown. †Untreated vs EGF-, $\mathrm{HC}$-, and $\mathrm{PGE}_{2}$-treated; $\ddagger \mathrm{EGF}$ vs $\mathrm{EGF}+\mathrm{HC}$ and $\mathrm{EGF}+\mathrm{HC}+\mathrm{Ind} ; * p<0.05$. (B) Morphology of the FHs74Int cells changed toward fibroblastoid during treatment with EGF and HC. Without any treatment, FHsInt74 cells were cuboidal and expressed cortical actin (white solid arrows). Upon EGF- and HC-treatment, they become elongated and actin was reorganized into stressfibers (open white arrowheads), which was inhibited by the addition of Ind.

pathologic processes during oncogenesis. Signaling cascade shared by EGF-R/COX-2 is a good example of this. Currently, both are implicated in malignant transformation and targeted in oncological clinical trials.

Based on our results, we conclude that concomitant use of Ind and $\mathrm{HC}$ in preterm infants may be harmful to the developing intestine if its synthesis of EGF-R ligands is poor. Production of EGF-family peptides correlates positively with gestation age and is unfortunately low in premature infants (34). Need for HC treatment reflects immaturity (35) and can thus be regarded as a further indicator of low EGF synthesis. If preterm infants develop intestinal problems, e.g. mucosal damage or ulceration, these lesions may not heal properly due to lack of mitogenic growth factors (e.g., EGF-R ligands) and poor mucosal defense. Conceivably, use of Ind and $\mathrm{HC}$ may interfere with $\mathrm{COX}-2 / \mathrm{PGE}_{2}$ production, which is already low due to insufficient production EGF-R ligands and 
predisposes the child to intestinal perforations. Carefully planned multicenter trials on EGF supplementation are needed.

\section{REFERENCES}

1. Watterberg KL, Gerdes JS, Cole CH, Aucott SW, Thilo EH, Mammel MC, Couser RJ, Garland JS, Rozycki HJ, Leach CL, Backstrom C, Shaffer ML 2004 Prophylaxis of early adrenal insufficiency to prevent bronchopulmonary dysplasia: a multicenter trial. Pediatrics 114:1649-1657

2. Attridge JT, Clark R, Walker MW, Gordon PV 2006 New insights into spontaneous intestinal perforation using a national data set: (2) two populations of patients with perforations. J Perinatol 26:185-188

3. Dollberg S, Lusky A, Reichman B 2005 Patent ductus arteriosus, indomethacin and necrotizing enterocolitis in very low birth weight infants: a population-based study. J Pediatr Gastroenterol Nutr 40:184-188

4. Fujii AM, Brown E, Mirochnick M, O’Brien S, Kaufman G 2002 Neonatal necrotizing enterocolitis with intestinal perforation in extremely premature infants receiving early indomethacin treatment for patent ductus arteriosus. J Perinatol 22:535-540

5. Grosfeld JL, Chaet M, Molinari F, Engle W, Engum SA, West KW, Rescorla FJ, Scherer LR 3rd 1996 Increased risk of necrotizing enterocolitis in premature infants with patent ductus arteriosus treated with indomethacin. Ann Surg 224:350-355

6. Miettinen PJ 1993 Transforming growth factor-alpha and epidermal growth factor in human fetal gastrointestinal tract. Pediatr Res 33:481-486

7. Wong WM, Wright NA 1999 Epidermal growth factor, epidermal growth factor receptors, intestinal growth, and adaptation. JPEN J Parenter Enteral Nutr 23:S83-S88

8. Miettinen PJ, Berger JE, Meneses J, Phung Y, Pedersen R, Werb Z, Derynck R 1995 Epithelial immaturity and multiorgan failure in mice lacking epidermal growth factor receptor. Nature 376:337-341

9. Dvorak B, Halpern MD, Holubec H, Williams CS, McWilliam DL, Dominguez JA, Stepankova R, Payne CM, McCuskey RS 2002 Epidermal growth factor reduces the development of necrotizing enterocolitis in a neonatal rat model. Am J Physiol Gastrointest Liver Physiol 282:G156-G164

10. Clark JA, Doelle SM, Halpern MD, Saunders TA, Holubec H, Dvorak K, Boitano SA, Dvorak B 2006 Intestinal barrier failure during experimental necrotizing enterocolitis: protective effect of epidermal growth factor treatment. Am J Physiol Gastrointest Liver Physiol 291:G938-G949

11. Leppa S, Saffrich R, Ansorge W, Bohmann D 1998 Differential regulation of c-Jun by ERK and JNK during PC12 cell differentiation. EMBO J 17:4404-4413

12. Abud HE, Watson N, Heath JK 2005 Growth of intestinal epithelium in organ culture is dependent on EGF signalling. Exp Cell Res 303:252-262

13. Sheng G, Bernabe KQ, Guo J, Warner BW 2006 Epidermal growth factor receptormediated proliferation of enterocytes requires p21waf1/cip1 expression. Gastroenterology 131:153-164

14. Salamanca CM, Maines-Bandiera SL, Leung PC, Hu YL, Auersperg N 2004 Effects of epidermal growth factor/hydrocortisone on the growth and differentiation of human ovarian surface epithelium. J Soc Gynecol Investig 11:241-251

15. Szabo IL, Pai R, Jones MK, Ehring GR, Kawanaka H, Tarnawski AS 2002 Indomethacin delays gastric restitution: association with the inhibition of focal adhesion kinase and tensin phosphorylation and reduced actin stress fibers. Exp Biol Med (Maywood) 227:412-424
16. Arsenault P, Menard D 1985 Influence of hydrocortisone on human fetal small intestine in organ culture. J Pediatr Gastroenterol Nutr 4:893-901

17. Ménard D, Arsenault P, Pothier P 1988 Biologic effects of epidermal growth factor in human fetal jejunum. Gastroenterology 94:656-663

18. Kajino H, Chen YQ, Chemtob S, Waleh N, Koch CJ, Clyman RI 2000 Tissue hypoxia inhibits prostaglandin and nitric oxide production and prevents ductus arteriosus reopening. Am J Physiol Regul Integr Comp Physiol 279:R278-R286

19. Bayer BM, Beaven MA 1979 Evidence that indomethacin reversibly inhibits cell growth in the G1 phase of the cell cycle. Biochem Pharmacol 28:441-443

20. Mammen JM, Matthews JB 2003 Mucosal repair in the gastrointestinal tract. Crit Care Med 31:S532-S537

21. Wells A, Harms B, Iwabu A, Koo L, Smith K, Griffith L, Lauffenburger DA 2006 Motility signaled from the EGF receptor and related systems. Methods Mol Biol 327:159-177

22. Uribe A, Johansson C 1988 Initial kinetic changes of prostaglandin E2-induced hyperplasia of the rat small intestinal epithelium occur in the villous compartments. Gastroenterology 94:1335-1342

23. Pai R, Soreghan B, Szabo IL, Pavelka M, Baatar D, Tarnawski AS 2002 Prostaglandin E2 transactivates EGF receptor: a novel mechanism for promoting colon cancer growth and gastrointestinal hypertrophy. Nat Med 8:289-293

24. Ali S, El-Rayes BF, Sarkar FH, Philip PA 2005 Simultaneous targeting of the epidermal growth factor receptor and cyclooxygenase-2 pathways for pancreatic cancer therapy. Mol Cancer Ther 4:1943-1951

25. Bailey JM, Makheja AN, Pash J, Verma M 1988 Corticosteroids suppress cyclooxygenase messenger RNA levels and prostanoid synthesis in cultured vascular cells Biochem Biophys Res Commun 157:1159-1163

26. Elder JB, Ganguli PC, Gillespie IE, Delamore WI, Gregory H 1975 Effect of urogastrone in the Zollinger-Ellison syndrome. Lancet 2:424-427

27. Gordon PV, Price WA, Stiles AD, Rutledge JC 2001 Early postnatal dexamethasone diminishes transforming growth factor alpha localization within the ileal muscularis propria of newborn mice and extremely low-birth-weight infants. Pediatr Dev Pathol 4:532-537

28. Warner BW, Erwin CR 2006 Critical roles for EGF receptor signaling during resection-induced intestinal adaptation. J Pediatr Gastroenterol Nutr 43:S68-S73

29. Bernal NP, Stehr W, Coyle R, Erwin CR, Warner BW 2006 Epidermal growth factor receptor signaling regulates $\mathrm{Bax}$ and $\mathrm{Bcl}-\mathrm{w}$ expression and apoptotic responses during intestinal adaptation in mice. Gastroenterology 130:412-423

30. Sigalet DL, Martin GR, Butzner JD, Buret A, Meddings JB 2005 A pilot study of the use of epidermal growth factor in pediatric short bowel syndrome. J Pediatr Surg 40:763-768

31. Clark JA, Lane RH, Maclennan NK, Holubec H, Dvorakova K, Halpern MD, Williams CS, Payne CM, Dvorak B 2005 Epidermal growth factor reduces intestinal apoptosis in an experimental model of necrotizing enterocolitis. Am J Physiol Gastrointest Liver Physiol 288:G755-G762

32. Walker-Smith JA, Phillips AD, Walford N, Gregory H, Fitzgerald JD, MacCullagh K, Wright NA 1985 Intravenous epidermal growth factor/urogastrone increases smallintestinal cell proliferation in congenital microvillous atrophy. Lancet 2:1239-1240

33. Sullivan PB, Brueton MJ, Tabara ZB, Goodlad RA, Lee CY, Wright NA 1991 Epidermal growth factor in necrotising enteritis. Lancet 338:53-54

34. Scott SM, Buenaflor GG, Orth DN 1989 Immunoreactive human epidermal growth factor concentration in amniotic fluid, umbilical artery and vein, serum, and placenta in full-term and preterm infants. Biol Neonate 56:246-251

35. Ng PC, Lee CH, Lam CW, Ma KC, Fok TF, Chan IH, Wong E 2004 Transient adrenocortical insufficiency of prematurity and systemic hypotension in very low birthweight infants. Arch Dis Child Fetal Neonatal Ed 89:F119-F126 